
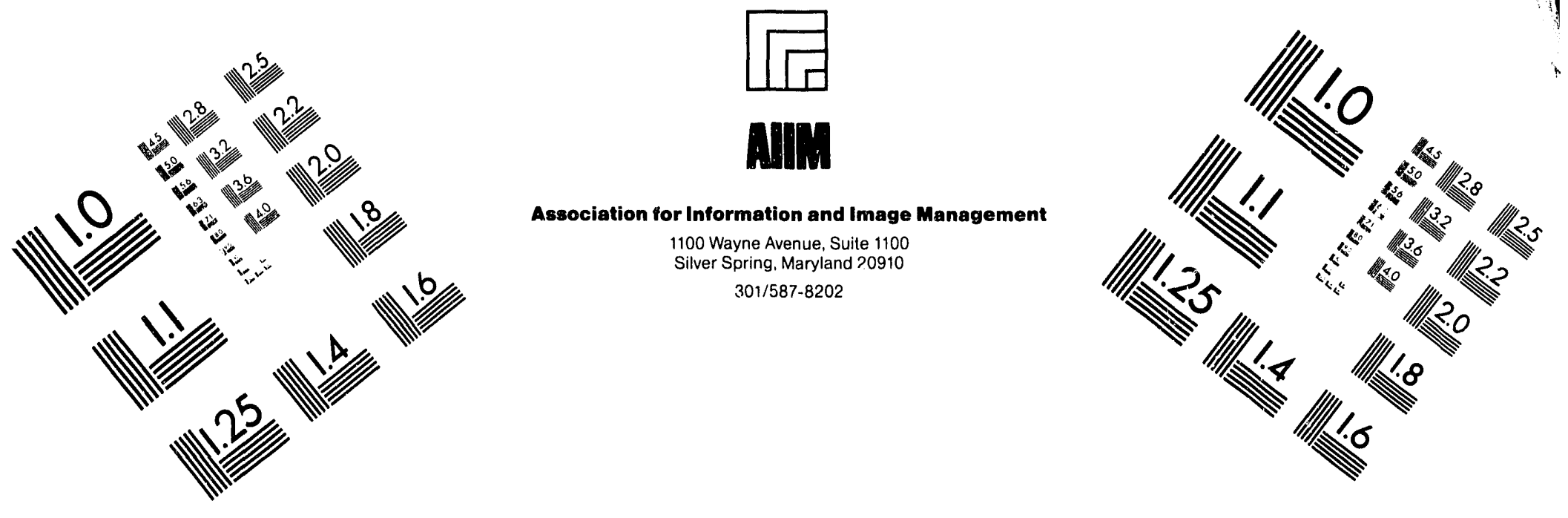

\title{
Centimeter
}

$\begin{array}{llllllllllllllll}1 & 2 & 3 & 4 & 5 & 6 & 7 & 8 & 9 & 10 & 11 & 12 & 13 & 14 & 15 & \mathrm{~mm}\end{array}$

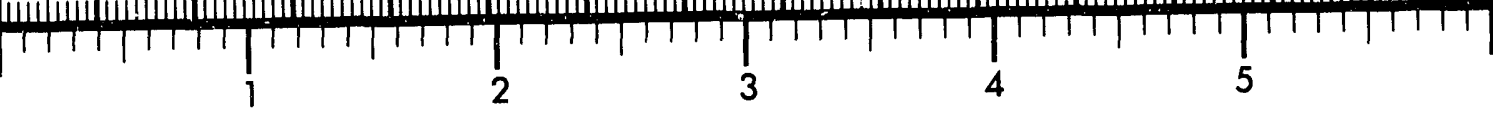
Inches
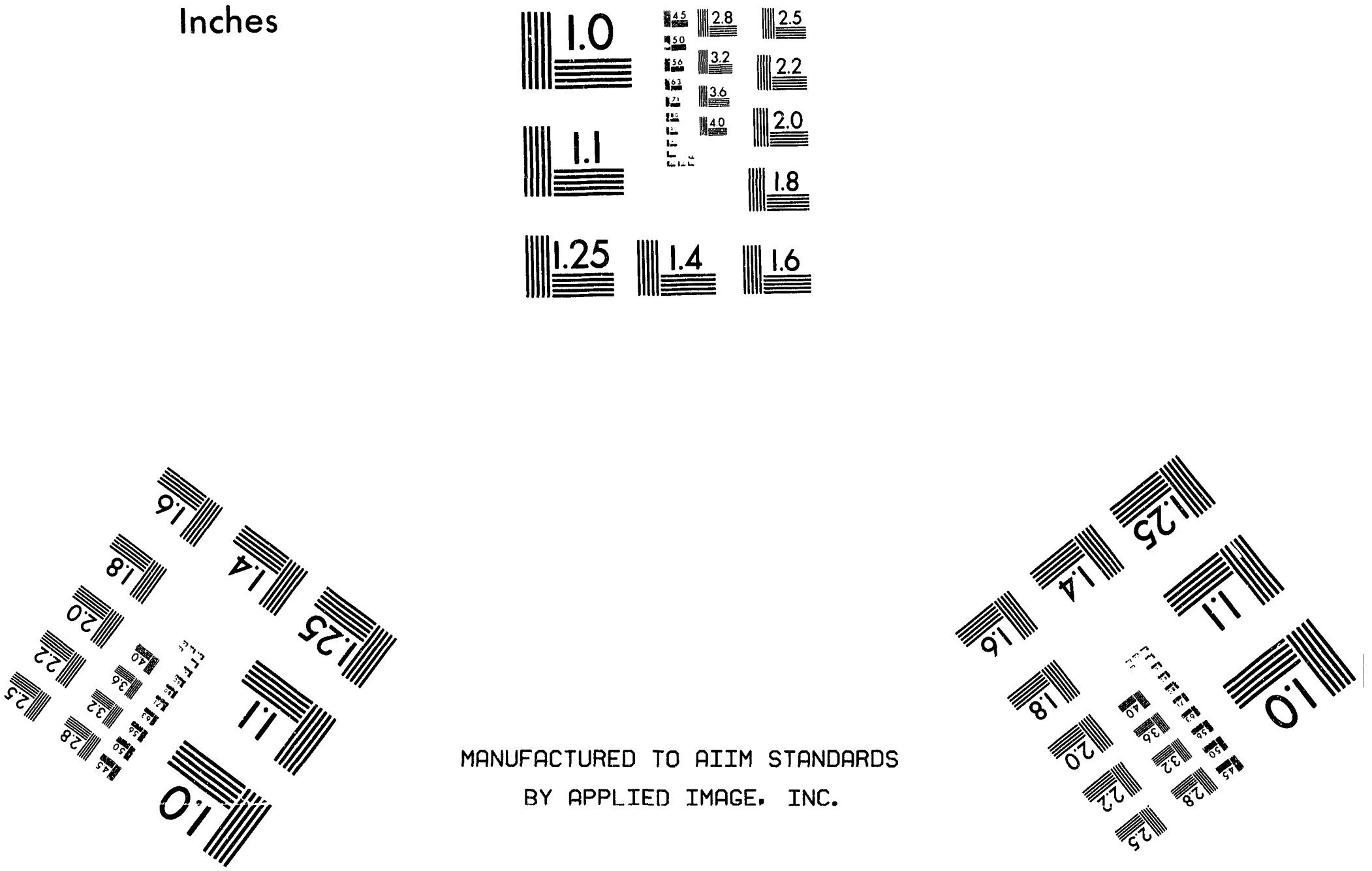

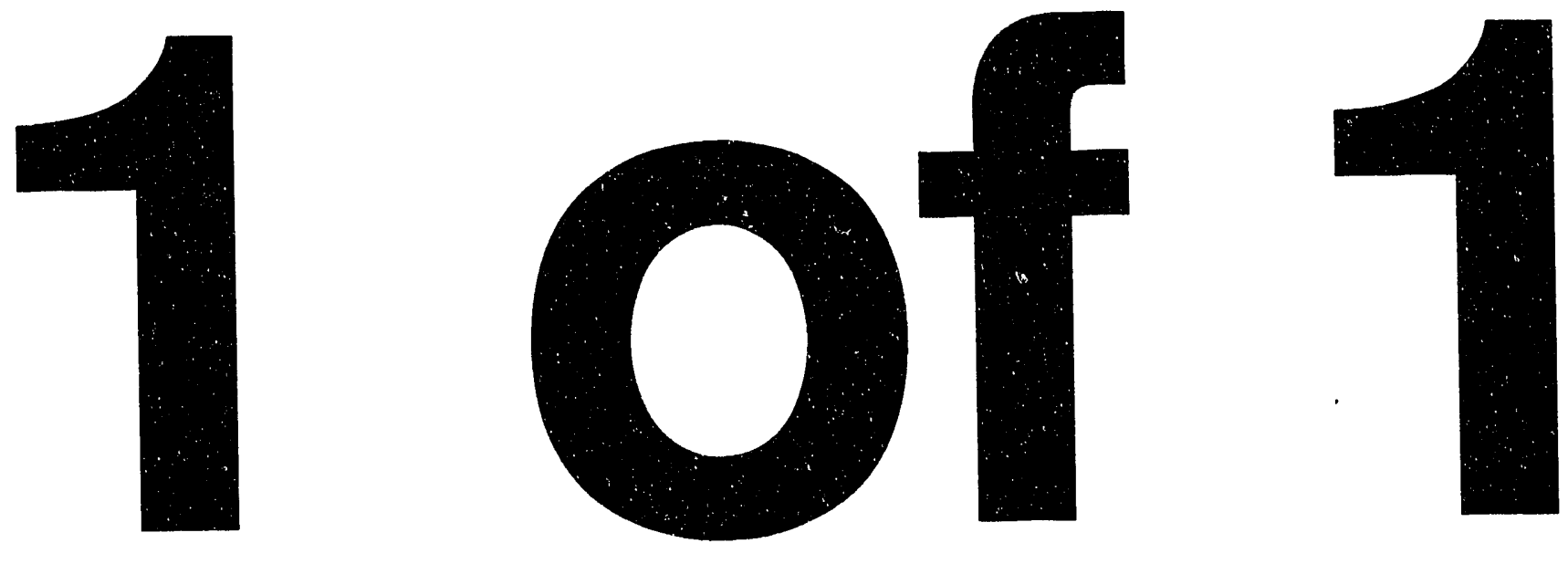


\title{
DOE/ER/40277-T)
}

DOE/ER/FINAL

\section{DIRECT MASS AND LIFETIME MEASUREMENTS OF NEUTRON- RICH NUCLEI UP TO A 100 USING THE TOFI SPECTROMETER AT LAMPF}

\author{
Final Report
}

for Period April 15, 1986 - March 14, 1992

V. Gordon Lind

Utah State University

Logan, Utah 84322

June 1993

Prepared for

THE U. S. DEPARTMENT OF ENERGY

AGREEMENT NO. DE-FG02-86ER40277

等 


\title{
Direct Mass and Lifetime Measurements of Neutron Rich Nuclei up to $A \sim 100$...
}

\author{
Final Scientific Report --- V. Gordon Lind, P. I.
}

--DOE grant \# 0-31722, Contract number DE-FG0286ER40277

\begin{abstract}
This project was directed toward the study of neutron-rich nuclei using the experimental facilities at LAMPF, which is a part of LANL. The principal results of the investigation includes the discovery of many new isotopes along with a measurement of their masses and in particular those nuclides in the $Z=7-19$ and $14-26$ regions of the chart of the nuclides. Thirty-four new nuclides were detected and studied with their masses being measured with relatively high accuracy and an additional twenty-six that were previously known and measured were remeasured to an improved accuracy. Besides providing new information about the mass surface in new and extended regions of the chart of the nuclides, this investigation enabled properties and previously unknown structure of some of the nuclei to be determined such as nuclear deformation among some of the nuclides. Also a study of the neutron pairing gaps and the proton pairing gaps among these nuclides was made. Other developments also achieved included instrument (TOFI) improvements and upgrades and theoretical investigations into the masses of the hadrons.
\end{abstract}

\section{PROJECTS AND EXPERIMENTS AT LAMPF USING TOFI}

Several experiments were conducted on the TOFI spectromenter during the period of this grant and USU was involved with most of them. In particular, two $\mathrm{Ph}$.D. level graduate students were heavily involved in the data acquired and analyzed for the $Z=7-19$ and 14 - 26 regions. These students used the work for their Ph.D. dissertations. They completed their degrees and now have post-doctoral positions at Texas A \& $\mathrm{M}$ in College Station, Texas. Also, data were obtained on TOFI on beta-delayed neutron and two neutron decay on many (about 40) ( her nuclei by a collaboration with Paul Reeder el al. of Pacific Northwest Laboratories to measure their lifetimes. USU personell participated somewhat in the collection of these data and are currently helping in the analysis. USU also supported a post-doctoral student who greatly helped in these experiments as well as being involved in an additional study of isomeric states in some of the same nuclei. 


\section{DATA ANALYSIS AND INTERPRETATION OF RESULTS}

The data for the studies of all nuclides between fluorine and chlorine were principally done by Xiao-Gang Zhou for his $\mathrm{Ph}$. D. dissertation. The masses of 34 neutron-rich isotopes were determined, ten, of which were first time determinations of these nuclides. The nuclei were produced by collisions between the energetic proton beam at LAMPF and heavy targets. The nuclei of interest were selected and passed through the TOFI spectromenter which separated them according to $\mathrm{M} / \mathrm{Q}$ (mass to charge ratio). Their atomic number was also measured using a Bragg spectrometer at the end of TOFI and by combining these results with the measured $M / Q$ the mass could be extracted. The results confirmed an anamoly of enhanced binding found in the sodium and magnesium isotopes that are very neutron-rich and also that the binding energy of these nuclei are less than previously reported. Careful comparisons with shell model calculations indicate that these results can be understood in terms of one or two neutron promotions from the sd shell to the fp shell. It appears that nuclei in the region of this anamoly can be described as an island of inversion where the nuclei are prolate deformed. For the nentron-rich isotopes of silicon through chlorine the nuclei seem to be well-behaved, i.e. they follow the expected behavior with no shell inversions.

Xiao-Lin Tu analyzed the $Z=17-26$ region for her dissertation, again studying the neutron-rich isotopes. In this experiment 28 new masses were measured for the first time and nine additional isotopes were measured with improved accuracy. Enhanced binding centered around scandium suggests the possibility of local deformation. The measurements also provided an investigation of the neutron and proton pairing energies as they depend on neutron-excess. The data show that a neutron excess $\left(\mathrm{I}^{2}\right)$ in the $\mathrm{Z}=21-23$ nuclei did make a differnce whereas there was little or no dependence upon neutron excess for the $Z=24-27$ region. It was also noted that the mass models tended to systematically overpredict the binding of neutron-rich $\mathrm{V}$ to $\mathrm{Fe}$ isotopes.

Other studies included removing the Bragg spectrometer and replacing it with neutron and beta detectors to measure the lifetimes of beta-delayed neutron and two-neutron decay. This was a collaboration between with P. L.Reeder, R. A. Warner, etc. of the Pacific Northwest Laboratories. This is ongoing work and Yi-Kyung Kim, a USU Ph.D. level student, is analyzing these data at the Pacific Northwest Laboratories as a part of his dissertation. Mr. Kim also designed and developed a velocity degrader technique for measuring $\mathrm{Z}$ which was placed in the TOFI line instead of using the Bragg spectrometer which made these lifetime measurements possible. He did this as his USU M.S. thesis work and was supported at that time by this grant. Work on isomeric states and support help for all the data taking and analysis came from Friedhelm Schreiber, a post-d octoral student from Germany, who was supported by this grant. Dr. Schreiber has now returned to his native country where he is gainfully employed. Finally, some theoretical studies on binding energies of various combinations of nucleons within the nucleus and a study of a systematic way of predicting the masses of the hadron elementary particles were conducted by the Gordon Lind, the P. I. and Jerry Long, an M. S. level graduate student working on the USU campus.

\section{BUDGET AND FUNDS EXPENDITURE}

The funds were spent as planned and budgeted. USU also contributed through partial support for Dr. Lind and some of the graduate students. 


\section{PUBLICATIONS AND SCIENTIFIC REPORTS}

- "Direct Mass Measurements of the Neutron-rich Isotopes of Cholrine Through Iron" S. L. Tu, S. G. Zhou, D. J. Vieira, J. M. Wouters, Z. Y. Zhou, H. L. Seifert, V. G. Lind, Zeitschrift fur Physik A. (Atomic Nuclei) 337, 361 (1990)

- "Exploration of the Neutron-rich Mass Surface from ${ }^{11} \mathrm{Li}$ to ${ }^{66} \mathrm{Fe} . " \mathrm{D}$. J. Vieira, X. G. Zhou, X. L. Tu, J. M. wouters, H. L. Siefert, K. E. IG. Lobner, Z. Y. Zhou, V. G. Lind, and G. N. Butler. Proc. International Workshop of "Nuclear Shapes and Nuclear Structure at Low Excitation Energies," Cargese, Corsica, June 3-7, 1991

- "Direct Mass Measurements of the Neutron-Rich Isotopes of Fluorine throught Chlorine", X. G. Zhou, X. L. Tu, J. M. Wouters, D. J. Vieira, D. E. G. Lobner, H. L. Seifert, Z. Y. Zhou and G. W. Butler, Physics Letters B 260 285-290 (1991)

- In addition to the above two student dissertations and one student M. S. thesis were written and microfilmed. They were:

1. "Direct Mass Measurements of Fluorine Through Chlorine Neutron-Rich Nuclei", Xiao-Gang Zhou, 1991

2. "Extension of the Mass Surface for Exotic Neutron-Rich Nuclei in the $Z=17-26$ Region", Xiao-Lin Tu, 1991

3. "Fast Ion Atomic Number Determination Using a Passive-Degrader Velocity Difference Technique", Yi-Kyung Kim, 1989

- In addition to the above, six talks were given at scientific mcetings. 

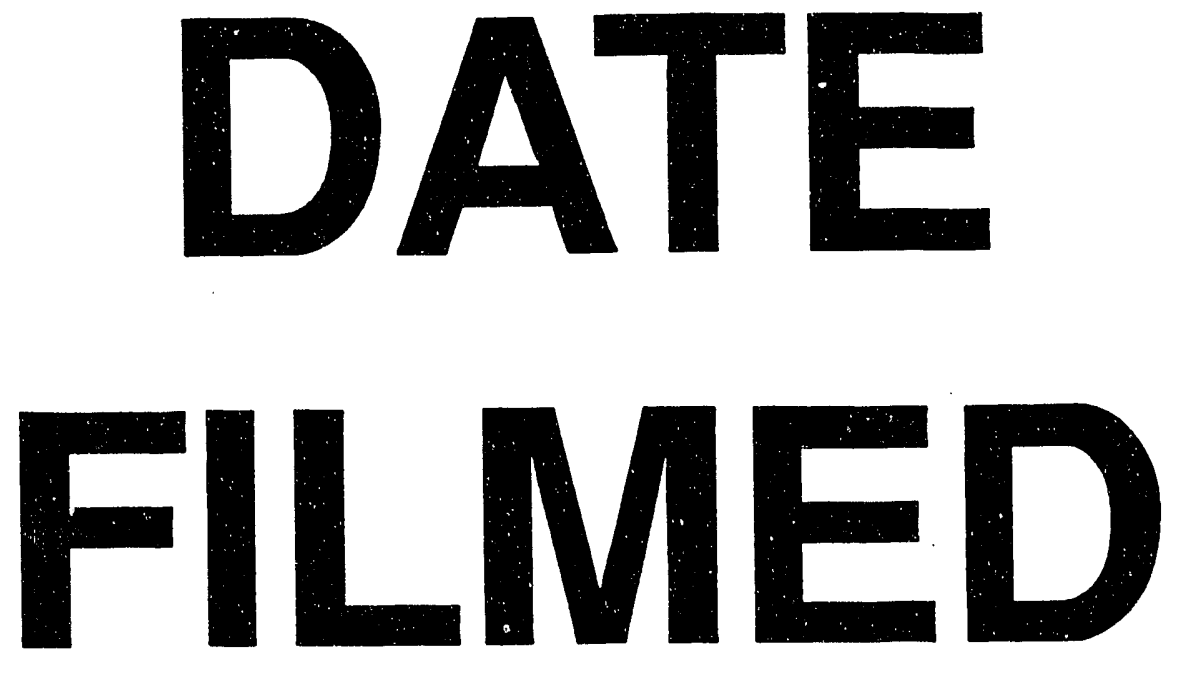

$8 / 17 / 93$
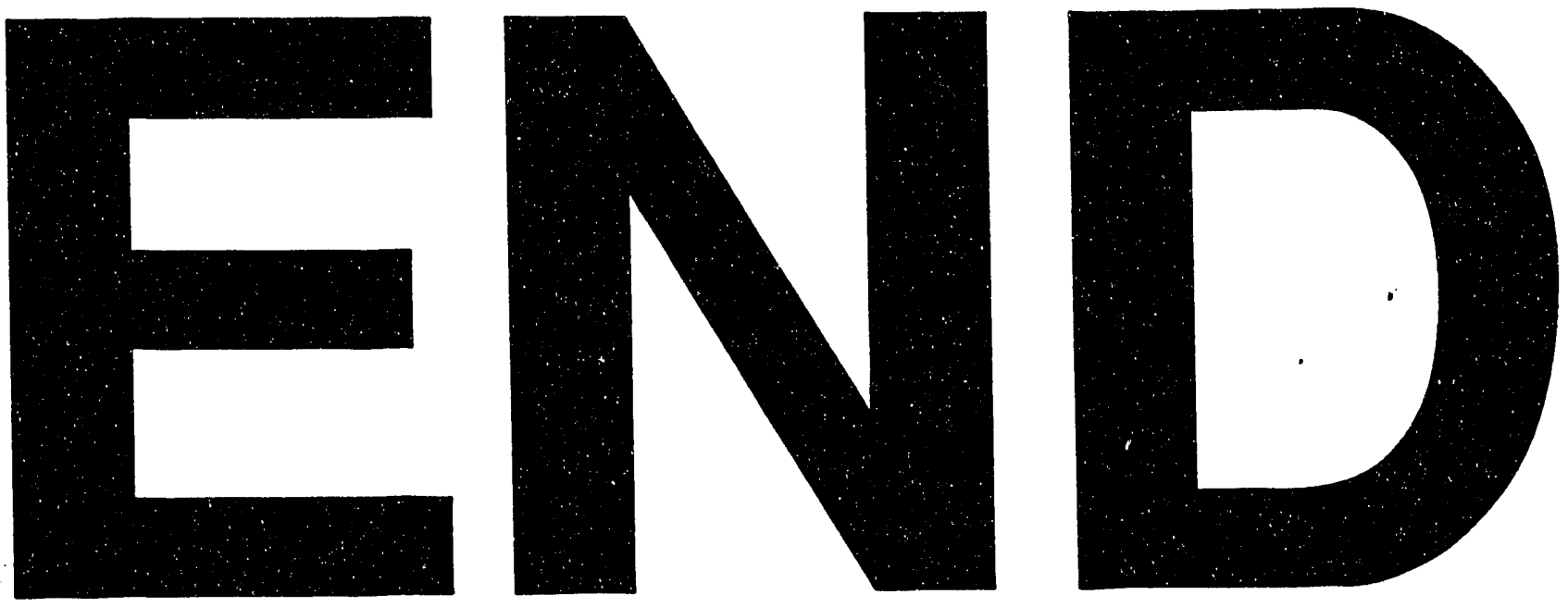
